\title{
Comments on New Research on Clinical Health Promotion
}

doi.org/10.29102/clinhp.19002

\section{Prevalence of limited health literacy among pa- tients with type 2 diabetes mellitus: A systematic review}

Abdullah A, Liew SM, Salim H, et al. PLoS ONE 2019;14:e0216402. doi.10.1371/ journal.pone.0216402

\section{Study description}

Diabetes type II increases all over the world, and living with diabetes requires a high degree of patient's empowerment and self-management that may be challenged by limited functional health literacy (F-HL). A new systematic review summarises the frequency of F-HL among patients with type II diabetes in different countries.

The study is based on 29 observational studies including 13,457 participants, mainly from the US, but also from Canada, Brazil, Switzerland, Netherlands, Marshall Island, South Korea and Taiwan. The quality of the studies ranges from moderate to good.

The results show a wide range in limited F-HL from $7.3 \%$ in Switzerland over $29 \%$ in the US to $82 \%$ in Taiwan. In addition, frequency also variated with the different tools used for measurement. Across the countries, the highest frequency of limited F-HL was seen in community populations and in population with shorter education.

\section{Comments from Professor Jürgen Pelikan}

There is a longer tradition in researching the relationship of diabetes self-management with health literacy, and there even has been developed a specific more comprehensive instrument for measuring diabetes health literacy. There also exists evidence that health literacy matters for self-management of diabetes. Surprising of the results of this review is that (functional) limited health literacy varies so much by country, but partly this seems to be a consequence of different instruments used for measuring (functional) health literacy in the included studies. Since there also has been demonstrated a social gradient for (functional) health literacy it is necessary to offer specific procedures for empowering diabetes patients for self-management that take into account limited health literacy of disadvantaged vulnerable patients. One way to achieve this would be to improve the organizational health literacy or health literacy sensitivity of health services and health professionals.

\section{A Randomized Trial of E-Cigarettes versus} Nicotine-Replacement Therapy

Hajek P, Phillips-Waller A, Przulj D, et al. N Engl J Med 2019;380:629-37. Doi: 10.1056/NEJMoa1808779

\section{Study description}

World-wide, smoking is still a major risk factor for increased morbidity and early mortality. There are two ways to reduce smoking; one aims not to start smoking at all and the other aims at successful quitting for those still smoking.

In a recent sizable randomised trial Hajek and colleagues compares e-cigarettes with nicotine replacement therapy (NRT) for smoking cessation. The study includes 886 participants, receiving either 3 months of NRT of their own preference or an e-cigarette starter pack with a refillable e-cigarette with nicotine e-liquid. All participants receive weekly behavioural support for at least 4 weeks.

The results show a significantly higher biochemically validated quit-rate after 1 year; $18 \%$ in the e-cigarette group compared to $10 \%$ in the NRT group. The frequency of side-effects is relatively high in both groups; significantly more in the e-cigarette group experiences mouth and throat irritation (65\% versus $51 \%$ ), while significantly more in the NRT group reports nausea, cough and phlegm. After one year, $80 \%$ of the quitters in the e-cigarette group still uses e-cigarettes compared to $9 \%$ using NRT in the NRT group.

\section{Comments from Professor Charlotta Holm Pisinger}

Professor and chair of the European Union Respiratory Society Charlotta Holm Pisinger (MD MPH PHD) from Copenhagen University comments that 'the results are in disagreement with two previous pragmatic randomized trials, which do not show significant effect on smoking cessation rates $(1 ; 2)$. Longitudinal real-life studies find that the use of e-cigarettes (EC) is associated with lower abstinence rates (3), and on population level the EC users (re)starting smoking after using ECs outnumber those who stop smoking after using ECs (4). The use of evidence-based pharmacotherapy and smoking cessation services has declined in Europe while use of ECs has increased (5), which is very worrying. Further, the authors haven't considered the growing body of evidence indicating negative health effects of longterm EC use $(6 ; 7) ; 80 \%$ of "smoke-free" participants assigned to ECs in the trial continued to use ECs - they still inhale toxicants and carcinogens. There is neither enough evidence supporting the safety of EC use nor their use as a smoking cessation tool." 


\section{Comments on New Research on Clinical Health Promotion}

\section{References}

(1) Halpern SD, Harhay MO, Saulsgiver K, et al. A Pragmatic Trial of E-Cigarettes, Incentives, and Drugs for Smoking Cessation. N Engl J Med 2018;378(24):2302-10. doi: 10.1056/NEJMsa1715757.

(2) Bullen C, Howe C, Laugesen M, et al. Electronic cigarettes for smoking cessation: a randomised controlled trial. Lancet 2013;382(9905):1629-37.

(3) Kalkhoran S, Glantz SA. E-cigarettes and smoking cessation in real-world and clinical settings: a systematic review and meta-analysis. Lancet RespirMed 2016;4(2):116-28.

(4) Liu X, Lugo A, Davoli E, et al. Electronic cigarettes in Italy: a tool for harm reduction or a gateway to smoking tobacco? Tob Control 2019 doi: 10.1136/tobaccocontrol-2018-054726.

(5) Filippidis FT, Laverty AA, Mons U, et al. Changes in smoking cessation assistance in the European Union between 2012 and 2017: pharmacotherapy versus counselling versus e-cigarettes. Tob Control 2018 doi: 10.1136/ tobaccocontrol-2017-054117.

(6) Pisinger C. A systematic review of health effects of electronic cigarettes: Prepared at the request of WHO Prevention of Noncommunicable Diseases, 2016.

(7) Prochaska JJ. The public health consequences of e-cigarettes: a review by the National Academies of Sciences. A call for more research, a need for regulatory action. Addiction 2018 doi: 10.1111/add.14478. 